\title{
Immediate-use steam sterilization sterilizes N95 masks without mask damage
}

\author{
Irene O. Carrillo MBA, MT(ASCP)CM, $\mathrm{CIC}^{1,2}$, Anna C.E. Floyd MPH, CIC, CHOP ${ }^{1,2}$, Christian M. Valverde MHA ${ }^{1,2}$, \\ Travis N. Tingle BSN, RN, CRCST ${ }^{1,2}$ and Firas R. Zabaneh MT, CIC, CIE, MBA ${ }^{1,2}$ \\ ${ }^{1}$ Houston Methodist Research Institute, Houston, Texas and ${ }^{2}$ Houston Methodist Hospital, Houston, Texas
}

To the Editor-As coronavirus disease 2019 (COVID-19) cases increase around the world, severe shortages of essential healthcare supplies are hampering the efforts to provide life-saving medical care without compromising the safety of healthcare workers. COVID-19 is transmitted by droplets, meaning that clinicians can be protected by gloves, gowns, eye shields, and masks. Disposable N95 masks seal tightly to the face and provide significantly better protection than surgical masks.

Established infectious disease protocol requires that clinicians dispose of N95 masks after contact with a patient. However, the United States will need 3.5 billion N95 masks for healthcare workers during this pandemic, and currently has $\sim 1 \%$ of that necessary volume. ${ }^{1}$ The Center for Disease Control and Prevention recommends that clinicians save masks and reuse them, and clinicians are currently using N95 masks for full shifts. ${ }^{2}$ With each attempt to safely don a contaminated N95 mask, the risk for infection of vital clinicians grows. In countries where equipment shortages have progressed, healthcare workers are currently being infected with SARS-CoV-2 at 3 times the rate of the general population, which reduces the ability of hospitals to provide adequate care and increases COVID-19 patient death rates. Thus, it is essential to create a protocol for sanitizing masks without reducing efficacy.

N95 masks are composed of thermoplastic elastomer (straps), aluminum (nose clip), polyurethane (nose foam), polypropylene (filter), and polyester (shell and cover web). The polyester shell and cover web are created from disorganized, thin fibers with an electrostatic charge. This design, while effective for reducing infection in clinicians, poses significant challenges for sanitization. Washing these masks with water decreased performance by $21 \%{ }^{3}$ Sanitizing N95 masks with alcohol similarly reduced performance by $37 \%$ and resulted in significant shrinkage. ${ }^{4}$ Ultraviolet germicidal irradiation has been tested for sanitization of N95 masks, but in $90 \%$ of cases, the integrity of the masks was compromised. ${ }^{5,6}$ Sanitization by bleach or ethylene oxide created significant risk to mask wearers due to residue left on the mask. ${ }^{6}$ Thus, novel methods of sanitizing N95 masks to ensure safety of clinicians working in factious disease units is needed.

Immediate-use steam sterilization (IUSS), using a Steris Amsco Evolution HC1500 PreVac Steam Sterilizer autoclave (Steris, Mentor, OH) was performed on N95 masks. Masks were packed in paper-plastic sterilization peel pouches for IUSS (Medical Action Industries 8" role, no. 422R). Masks were photographed

Author for correspondence: Firas R. Zabaneh, E-mail: fzabaneh@houstonmethodist.org Cite this article: Carrillo IO, et al. (2020). Immediate-use steam sterilization sterilizes N95 masks without mask damage. Infection Control \& Hospital Epidemiology, 41: 1104-1105, https://doi.org/10.1017/ice.2020.145

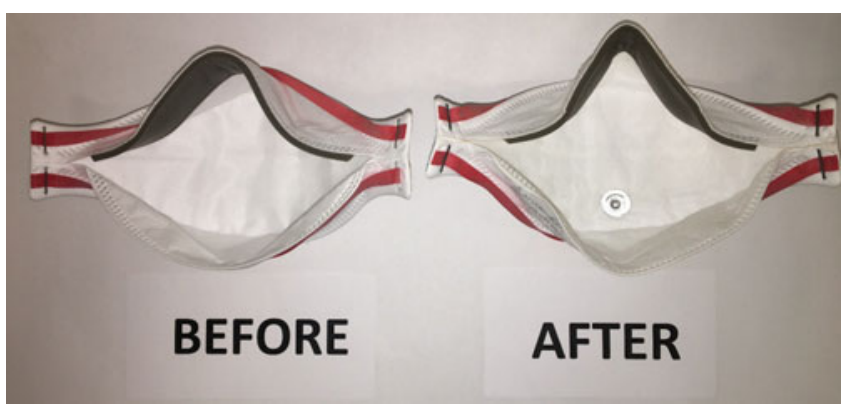

Fig. 1. N95 masks retained structural integrity after 3 immediate-use steam sterilization (IUSS) cycles.

and fit tested prior to IUSS, and this testing protocol was repeated after the IUSS cycle. The 3M 1870 and M3 1870+ masks (3M, Saint Paul, MN) retained efficacy in a quantitative fit test. Quantitative fit tests were performed using the gold standard TSI PortaCount Respirator Fit Tester (TSI, Shoreview, MN).

We tested 5 subjects to investigate individual differences between faces. For each subject, a fit test was performed before the IUSS cycle as a control. Fit tests were performed again after 3 IUSS cycles. In all cases, masks retained their structural integrity and efficacy (Fig. 1).

The IUSS cycle is performed with a chemical indicator $(3 \mathrm{M}$ Comply SteriGage Steam Chemical Integrator, no. 422R) and a biological indicator (3M Attest Super Rapid 5 Steam Plus Callenge Pack, no. 41482V) for every autoclave cycle, confirming that no biological or chemical contamination is present on the masks. If either indicator fails the IUSS cycle, the masks are reprocessed and are not placed into the hospital system.

Our stuy was limited by a small sample size. Follow-up studies will be conducted with a significantly larger sample by recruiting participants from the Houston Methodist Hospital who will be fit tested for their mask each day.

Despite the limitations of this study, the data herein provide a valid basis for the use of IUSS for N95 masks to prevent the spread of SARS-CoV-2 to healthcare workers.

\section{References}

1. US Congress. Committee on Appropriations, Subcommittee on the Departments of Labor, Health and Human Services, Education, and Related Agencies. Budget hearing on the Department of Health and Human Services, February 25, 2020. FDCH Political Transcripts.

2. Strategies for optimizing the supply of N95 respirators: COVID-19. Centers for Disease Control and Prevention website. https://www.cdc.gov/coronavirus/ 2019-ncov/hcp/respirators-strategy/contingency-capacity-strategies.html Published 2020. Accessed March 24, 2020. 
3. Viscusi DJ, King WP, Shaffer RE. Effect of decontamination on the filtration efficiency of two filtering facepiece respirator models. J Int Soc Respir Prot 2007;24:93-107.

4. Martin SB, Mayor ES. 33. Electrostatic respirator filter media: filter efficiency and most penetrating particle size effects. Appl Occup Environ Hyg 2000; 15:609-617.
5. Lindsley WG, Martin SB, Thewlis RE, et al. Effects of ultraviolet germicidal irradiation (UVGI) on N95 respirator filtration performance and structural integrity. J Occup Environ Hyg 2015;12:509-517.

6. Lindsley WG, Martin SB, Thewlis RE, et al. Evaluation of five decontamination methods for filtering facepiece respirators. Ann Occup Hyg 2009; 12:509-517.

\title{
Environmental cleaning is effective for the eradication of severe acute respiratory syndrome coronavirus 2 (SARS-CoV-2) virus in contaminated hospital rooms: A patient from the Diamond Princess cruise ship
}

\author{
Yosuke Hirotsu $\mathrm{PhD}^{1}$ (1), Makoto Maejima BS², Masumi Nakajima BN ${ }^{3}$, Hitoshi Mochizuki MD ${ }^{1,4}$ and Masao Omata MD ${ }^{4,5}$ \\ ${ }^{1}$ Genome Analysis Center, Yamanashi Central Hospital, Kofu, Yamanashi, Japan, ${ }^{2}$ Division of Microbiology in Clinical Laboratory, Yamanashi Central Hospital, \\ Kofu, Yamanashi, Japan, ${ }^{3}$ Division of Infection Disease Control, Yamanashi Central Hospital, Kofu, Yamanashi, Japan, ${ }^{4}$ Department of Gastroenterology, \\ Yamanashi Central Hospital, Kofu, Yamanashi, Japan and ${ }^{5}$ The University of Tokyo, Tokyo, Japan
}

To the Editor-Doctors, nurses, and other medical staff are greatly concerned about nosocomial outbreaks of severe acute respiratory syndrome coronavirus 2 (SARS-CoV-2). Environmental contamination is a possible source of nosocomial transmission., ${ }^{1,2}$ However, how effective environmental cleaning is against SARS-CoV-2 remains unclear.

A 75-year-old man infected with SARS-CoV-2 was diagnosed with COVID-19 during the quarantine period on the Diamond Princess cruise ship. He was transferred directly to our hospital on February 11, 2020. He resided in patient room A for 2 days then was moved to room B, where he stayed for 19 days. After cleaning the rooms thoroughly with disinfectant $\left(\right.$ Rely $^{+}$On Virkon, LANXESS, or RUBYSTA in Japan), we tested 15 areas that were in close contact with the patient and medical staff. Swabs were used to transfer 5 environmental samples from room A and 10 samples from room B to universal transport media (Copan, Murrieta, CA). Cleaning was conducted immediately after the patient left the rooms. Environmental sampling was conducted within 5 days and $30 \mathrm{~min}$ after the patient left rooms $\mathrm{A}$ and $\mathrm{B}$, respectively. Nucleic acids were extracted using MagMAX Viral/Pathogen Nucleic Acid Isolation Kit (ThermoFisher Scientific, Waltham, $\mathrm{MA}$ ) and were tested using real-time reverse transcription polymerase chain reaction (RT-PCR) targeting the nucleocapsid $(N)$ gene of SARS-CoV-2. Seven sets of primers and probes (CDC-N1, CDC-N2, CDC-N3, YCH-N1, YCH-N2, NIID-N1, and NIID-N2) were used to detect SARS-CoV-2 as previously described (Supplemental Table 1 online). ${ }^{3}$ For the internal positive control, the human ribonuclease $\mathrm{P} 30$ subunit ( $R P P 30$ ) gene was used. The patient's records, timing of cleaning and sampling, and RT-PCR results were collated.

Author for correspondence: Yosuke Hirotsu, E-mail: hirotsu-bdyu@ych.pref. yamanashi.jp

Cite this article: Hirotsu Y, et al. (2020). Environmental cleaning is effective for the eradication of severe acute respiratory syndrome coronavirus 2 (SARS-CoV-2) virus in contaminated hospital rooms: A patient from the Diamond Princess cruise ship. Infection Control \& Hospital Epidemiology, 41: 1105-1106, https://doi.org/10.1017/ ice.2020.144
On admission, the patient had fever $\left(39^{\circ} \mathrm{C}\right)$ and a mild cough (Supplemental Table 2 online). The chest X-ray and computed tomography scan on day 1 showed signs of pneumonia in both lungs. He received lopinavir/ritonavir and antibacterial therapy on day 2, but showed respiratory failure. He received supplemental oxygen from day 4 to day 15 . After careful clinical management, the patient's overall status improved. RT-PCR showed that his sputum was positive for SARS-CoV-2 on day 11. Subsequently, nasopharyngeal swabs were negative on days 17, 22, and 29.

The patient stayed in room A for 3 days, during which he had the SARS-CoV-2 infection. After cleaning room A, 5 environmental samples were examined by RT-PCR. All samples were negative for SARS-CoV-2 and were positive or negative for RPP30 (Table 1).

After the patient left room $A$, he resided in room $B$ for 20 days. Ten environmental samples were collected after cleaning. All 10 samples from room $B$ were negative for SARS-CoV-2 and were positive or negative for RPP30 (Table 1 ).

SARS-CoV-2 is detectable in several types of clinical samples including bronchial lavage fluid, nasopharyngeal swab, pharyngeal swab, sputum, saliva, and feces. ${ }^{4,5}$ Transmission of SARS-CoV-2 via surfaces in hospitals is of great concern to medical staff and patients. Blocking the potential routes of transmission is essential for preventing the spread of SARS-CoV- $2 .{ }^{6}$ A recent study showed that environmental contamination can occur via contact with patients with SARS-CoV-2 and upper respiratory tract symptoms. ${ }^{7}$ After cleaning, all areas were negative for SARS-CoV-2; therefore, thorough cleaning is sufficient for SARS-CoV-2 decontamination.

This study had several limitations. First, RT-PCR was not performed before cleaning because of the risk of nosocomial transmission. Therefore, a comparison of the viral loads of high-touch areas before and after cleaning is required. Second, this study involved a single patient, and further studies are required to confirm these findings.

In summary, our data indicate the effectiveness of environmental cleaning for SARS-CoV-2 decontamination. This information is useful for infection control strategies and may alleviate the concerns of medical staff. 